\title{
Continuous Phase Shift of Sinusoidal Signals Using Injection Locked Oscillators
}

\author{
J. M. López-Villegas, Senior Member, IEEE, J. G. Macias, J. A. Osorio, J. Cabanillas, Member, IEEE, \\ J. J. Sieiro, Member, IEEE, J. Samitier, Associate Member, IEEE, and N. Vidal
}

\begin{abstract}
This work presents an alternative to generate continuous phase shift of sinusoidal signals based on the use of super harmonic injection locked oscillators (ILO). The proposed circuit is a second harmonic ILO with varactor diodes as tuning elements. In the locking state, by changing the varactor bias, a phase shift instead of a frequency shift is observed at the oscillator output. By combining two of these circuits, relative phases up to $\pm 90^{\circ}$ could be achieved. Two prototypes of the circuit have been implemented and tested, a hybrid version working in the range of $200-300 \mathrm{MHz}$ and a multichip module (MCM) version covering the 900-1000 $\mathrm{MHz}$ band.
\end{abstract}

Index Terms - CMOS analog integrated circuits (ICs), injection locked oscillators (ILOs), multichip modules (MCMs), phase shifters.

\section{INTRODUCTION}

$\mathbf{P}$ HASE Shifting is a key issue in modern communication systems. Beam steering and beam forming in phase array antennas, image rejection mixers, carrier recovery circuits or I/Q modulation and demodulation, are examples where signals with a precise phase shift are required. Different topologies of phase shifters have been reported in the literature. For fixed amounts of phase shift (i.e., multiples of $90^{\circ}$ ), poly-phase networks are the preferred solutions [1]. When a digitally controlled phase shift is required, phase shifters based on PIN or varactor diodes and FET transistors are commonly used [2].

Another way to obtain sinusoidal signals with a precise phase displacement is direct generation. Instead of having a signal source connected to a phase shifter, the required signals are generated using a multiphase oscillator. Cross-coupled oscillators and ring oscillators are examples of multiphase output circuits [3]. In this case, a signal is forced to follow a closed loop and the phase shift is accomplished by sampling the signal at the right place. Depending on the loop elements different fixed values of the phase shift can be achieved.

Sub-harmonic injection locking of oscillator circuits has also been proposed as an alternative method to generate continuous phase displacement of a sinusoidal signal [4]. Nevertheless, this

\footnotetext{
Manuscript received May 11, 2004. revised February 11, 2005. This work was supported in part by the Spanish Ministry of Science and Technology under project TIC2001-2947-C02-01. The review of this letter was arranged by Associate Editor J.-G. Ma.

J. M. López-Villegas, J. G Macias, J. A. Osorio, J. Cabanillas, J. J. Sieiro, and J. Samitier are with the Department of Electronics, Instrumentation and Communication Systems, RF Group, University of Barcelona, Barcelona E-08028, Spain (e-mail: josem@el.ub.es).

N. Vidal is with Escola Universitaria Salesiana de Sarrià (EUSS), Barcelona E-08017, Spain (e-mail: nvidal@euss.es ).

Digital Object Identifier 10.1109/LMWC.2005.847690
}

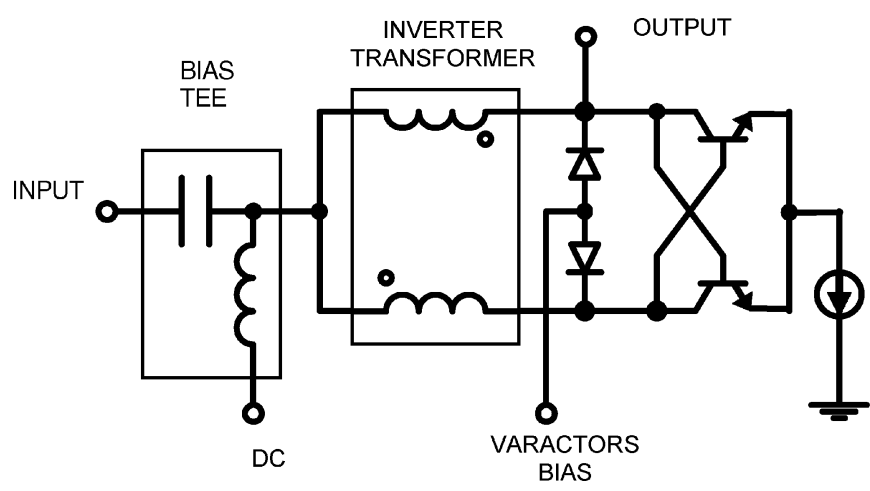

Fig. 1. Circuit schematic of second harmonic ILO with varactor control.

method implies a phase noise degradation of the output signal, when compared with the injected one. In this work an alternative scheme of phase shifting is reported. It is based on the phase behavior of second harmonic ILOs [5]. When compared with sub-harmonic injection, the proposed method allows continuous phase shift in a shorter range, but improves the phase noise behavior. Consequently, the use of sub or super harmonic injection will depend on the trade-off between the requirements of phase tuning and phase noise.

\section{Circuit Fundamentals}

Injection is an usual way to synchronize an oscillator with an incident signal. When the injected signal is close to a harmonic of the oscillator free running frequency, the ensemble is known as a super-harmonic ILO [5].

A possible implementation of second harmonic ILO is shown in Fig. 1. The circuit is a cross pair oscillator, whose resonant tank consists of an inverter transformer (inductive element) and a pair of varactors. The second harmonic is injected at the centre tap of the transformer. Ideally, under common mode excitation, the transformer acts as a short circuit, thus the injected signal is found without distortion at the varactors' terminals. There, due to the non linear behavior of the stored charge versus applied voltage, the injected signal at frequency $2 f_{l}$ mixes with the oscillator signal at frequency close to $f_{l}$. First-order harmonic analysis gives for these signals

$$
V_{1}=A_{1} \cos \left(2 \pi f_{l} t+\Phi(t)\right) ; \quad V_{2}=A_{2} \cos \left(4 \pi f_{l} t+\psi\right)
$$

being $V_{1}$ and $V_{2}$ the fundamental component of the oscillator voltage and the injected signal, respectively, $A_{1}$ and $A_{2}$ their amplitudes and, $\Phi(t)$ and $\psi$ the corresponding phases. Note that phase $\Phi(t)$ takes into account the dynamics of the oscillator 


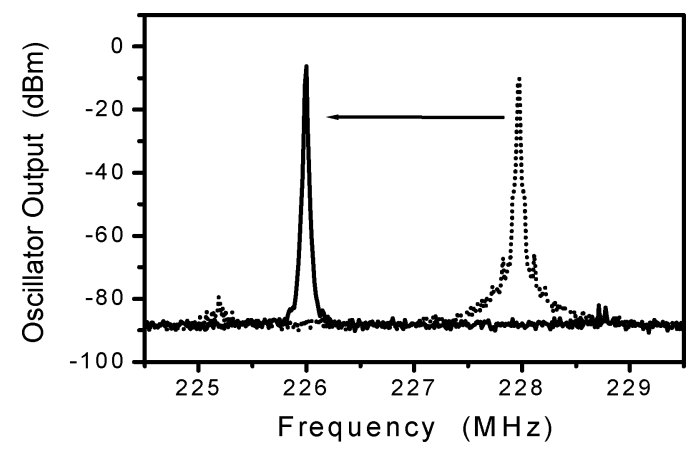

Fig. 2. Measured output spectrum of a second harmonic ILO: dotted line is the free running oscillation, continuous line is the forced oscillation when a $452-\mathrm{MHz}$ signal is injected at the input.

frequency $f(t)$ from the free running state, $f_{r}$, to the locked state, $f_{l}$ (i.e $f(t)=f_{l}+d \Phi(t) / d t$ ).

By defining angle $\theta(t)=2 \Phi(t)-\psi-\pi / 2$, and after some trigonometric calculations, the fundamental current $I_{1}$, passing through the varactor diodes can be expressed as

$$
I_{1}=\left(C_{D C}-\alpha \frac{A_{2}}{2} \sin \theta(t)\right) \frac{d V_{1}}{d t}+\alpha A_{2} \pi f_{l} \cos \theta(t) V_{1}
$$

where $C_{D C}$ and $\alpha$ are the capacitance of the varactors and its derivative versus the applied voltage, respectively, both at the bias point $V_{D C}$.

According to (2), the varactors' capacitance $C(\theta)$ (i.e., term between brackets) changes depending on the amplitude $A_{2}$ and angle $\theta(t)$. As a consequence there is a change of the oscillator frequency after the injection of the input signal. This change is reflected in $\Phi(t)$ and also in $\theta(t)$. Equilibrium is reached when $d \theta(t) / d t=0$. Accordingly, the time derivative of $\Phi(t)$ should also be zero and then, the final oscillator's frequency will be the locking frequency $f_{l}$. An example of this frequency synchronization is shown in Fig. 2.

At the steady-state the oscillation frequency can be expressed as follows:

$$
f_{l}=\frac{1}{2 \pi \sqrt{L C_{s s}(\theta)}}
$$

where $C_{s s}(\theta)$ is the steady state capacitance. By substituting in (3) the term between brackets in (2), the steady state value for angle $\theta$ can be found

$$
\theta_{s s}=\arcsin \left(\frac{2 C_{D C}}{\alpha A_{2}} \frac{f_{l}^{2}-f_{r}^{2}}{f_{l}^{2}}\right) .
$$

According to the above analysis the phase shift process can be explained as follows. Let us consider a second harmonic ILO in the locked state. The oscillator output frequency is $f_{l}$ and angle $\theta(t)$ is equal to $\theta_{s s}$. At this point let us consider the effect of a modification in the varactors bias. The capacitance $C_{D C}$, its derivative $\alpha$ and the free running frequency $f_{r}$ will change. Provided these changes are small (in practice the argument of the arcsin function in (3) must be kept between -1 and 1), the output frequency will still be $f_{l}$, and a new steady state condition will be reached, corresponding to a new value of $\theta_{s s}$. Finally,

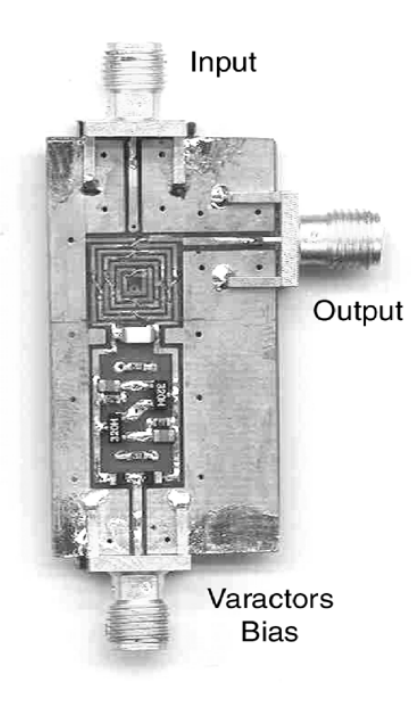

(a)

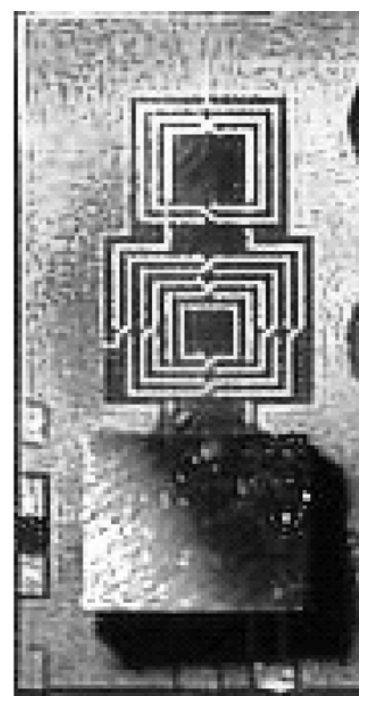

(b)
Fig. 3. Photographs of the second harmonic ILO circuits: (a) hybrid version and (b) MCM version.

the modification of angle $\theta_{s s}$ before and after the bias change, $\Delta \theta_{s s}$ implies a change in the output phase $\Delta \Phi=\Delta \theta_{s s} / 2$. This relationship between phases also explains the phase noise improvement in super-harmonic ILO's [5].

\section{Circuit Design AND FABRICATION}

Two different versions of the second harmonic ILO circuit have been fabricated and tested. First one is a hybrid version working in the $200-300 \mathrm{MHz}$ frequency band. The second is a MCM version in the $900 \mathrm{MHz}-1 \mathrm{GHz}$ frequency band (Fig. 3).

The hybrid version has been implemented using lumped components on a printed circuit board. Transformers have been printed directly on board as interleaved square spirals. Electromagnetic simulations tools have been used to optimize their geometry in order to achieve good performance in both, common and differential modes of operation.

The MCM version has been implemented using a Pyrex 7740 substrate carrier. Two $1.5-\mu \mathrm{m}$ thick $\mathrm{Al}$ metal levels are used as interconnects and to perform the required integrated transformers. Polyimide, $4.5-\mu \mathrm{m}$ thick, is used as intermetal dielectric and passivation layer. RFIC's die including the active part of the oscillator has been fabricated using a $0.35-\mu \mathrm{m}$ CMOS process. This die has been flip-chipped on the carrier substrate using $\mathrm{Pb} / \mathrm{Sn}$ solder bumps over pads with a previous $\mathrm{Ti} / \mathrm{Ni} / \mathrm{Au}$ metallization.

\section{EleCtrical CHARACTERIZATION}

In order to accurately measure the phase shift, two second harmonic ILO circuits have been injected with the same input signal. One of them is used as a reference while the other is used as a test circuit. The output of the reference circuit is the trigger for measuring the phase shift of the test circuit. Fig. 4 shows the obtained results for the hybrid version. Waveforms corresponding to the reference circuit (bottom) and the test circuit under three varactor bias conditions (top) are plotted. A $444-\mathrm{MHz}-6 \mathrm{dBm}$ input signal is injected to both second 


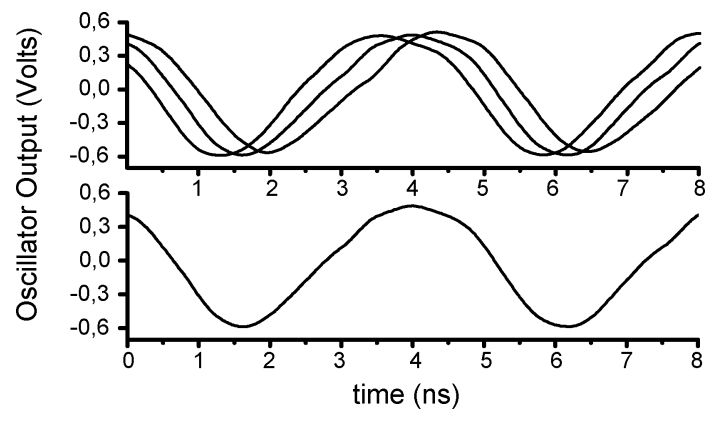

Fig. 4. Measured output waveforms of the reference oscillator circuit (bottom) and test circuit for three varactor bias conditions (top).

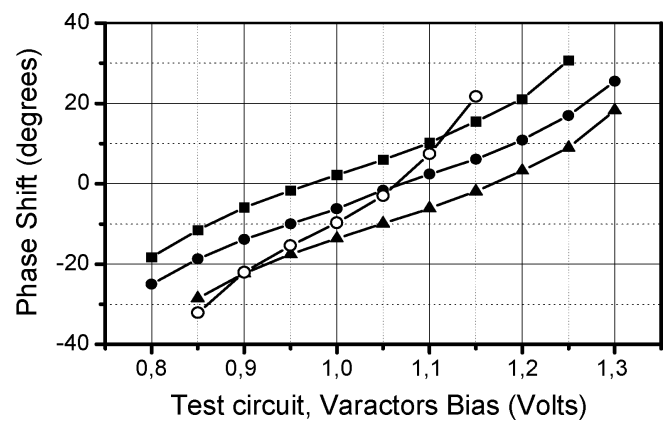

Fig. 5. Phase shift at test circuit output as a function of the varactors bias. Outlined data points $(O)$ correspond to $3 \mathrm{dBm}$ injected power and $1.1 \mathrm{~V}$ for the reference circuit varactors bias (Vref). Filled points correspond to 6-dBm injected power and Vref: (ם) $1 \mathrm{~V},(\bullet) 1.1 \mathrm{~V}$ and $(\boldsymbol{\Delta}) 1.2 \mathrm{~V}$.

harmonic ILOs resulting in an output locking frequency of $222 \mathrm{MHz}$.

In Fig. 5, the phase shift between the outputs of the test and reference circuits is plotted as a function of the varactors bias of the test circuit. Four different sets of phase/bias values are represented corresponding to different values of the reference circuit varactors bias and the injected power. Ideally, the achievable phase shift ranges from $-90^{\circ}$ to $90^{\circ}$. Any further intent to increase the phase shift would unlock one or both second harmonic ILOs. In practice, the useful phase shift range is more restricted due to inaccuracies in the phase control. According to expression (4) the phase sensitivity is, in a first approach, inversely proportional to the injected amplitude $A_{2}$. Consequently, the phase-shift/Bias slope should decrease as the injected power increases. This fact is clearly observed in Fig. 5. Finally, particularly interesting is the existence of a range of phase/bias values showing a nearly linear behavior, which will allow direct analogue phase modulation.

In order to investigate the dynamic behavior of the phase shifting process, the outputs of the reference and test circuits have been added to perform a phase modulation (PM) to amplitude modulation (AM) conversion. The process is depicted schematically in Fig. 6. The output phasor of the reference ILO is combined with the output phasor of the test ILO for two different varactor biases. The resultant phasor changes its amplitude according to the phase shift of the test ILO. Fig. 7 shows the measured amplitude modulation pattern obtained using this procedure with two MCM second harmonic ILOs. The varactor bias for one of them is kept constant while for the other it changes ac-

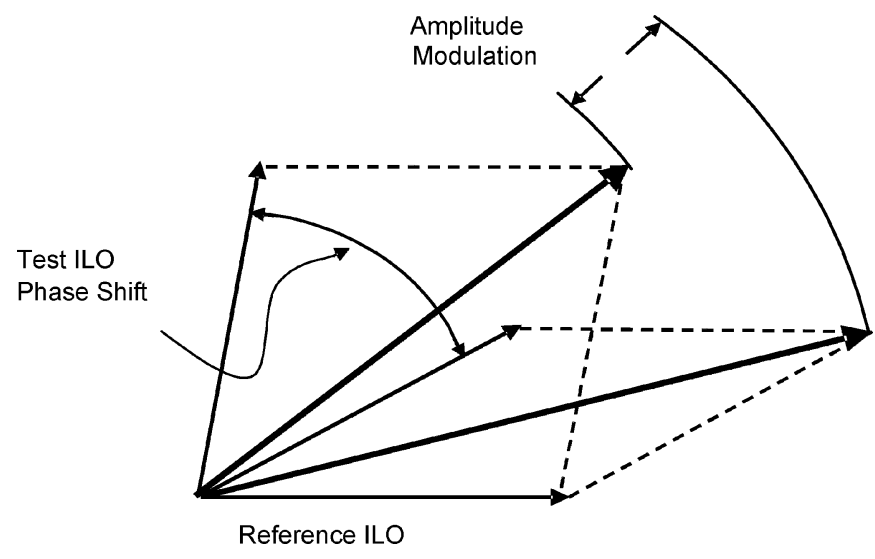

Fig. 6. Phasor diagram showing the amplitude modulation resulting after adding the test and reference ILO's outputs.

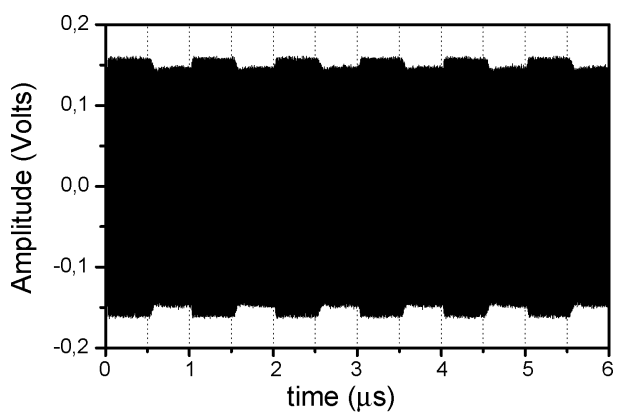

Fig. 7. Amplitude modulation pattern generated adding the outputs of two MCM second harmonic ILO circuits.

cording to a 1-MHz square wave. In this case the output locking frequency is $940 \mathrm{MHz}$.

\section{CONCLUSION}

Continuous phase shifting using second harmonic Injection locked oscillators have been demonstrated in this work. Both static and dynamic phase shift behaviors have been investigated using two circuit prototypes. One hybrid version and a MCM version intended to work in the frequency ranges of 200-300 MHz and $900 \mathrm{MHz}-1 \mathrm{GHz}$, respectively. The proposed circuits could be used as a direct narrow band analog or digital phase modulator.

\section{REFERENCES}

[1] M. Borremans, B. De Muer, and M. Steyaert, "The optimization of $\mathrm{GHz}$ integrated CMOS quadrature VCO's based on a poly-phase filter loaded differential oscillator," in Proc. IEEE Int. Symp. Circuits Systems (ISCAS'00), 2000, pp. II-729-II-732.

[2] R. Tang and R. W. Burns, "Array technology," Proc. IEEE, vol. 80, pp. 173-182, Jan. 1992.

[3] J. van der Tang, P. van de Ven, D. Kasperkovitz, and A. van Roermund, "Analysis and design of an optimally coupled 5-GHz quadrature LC oscillator," IEEE J. Solid-State Circuits, vol. 37, no. 5, pp. 657-661, May 2002.

[4] X. Zhang and A. S. Daryoush, "Full $360^{\circ}$ phase shifting of injectionlocked oscillators," IEEE Microw. Guided Wave Lett., vol. 3, no. 1, pp. 14-16, Jan. 1993.

[5] H. R. Rategh and T. H. Lee, "Superharmonic injection-locked frequency dividers," IEEE J. Solid-State Circuits, vol. 34, no. 6, pp. 813-821, Jun. 1999. 